\title{
Resonant scattering probes for terahertz near-field microscopy
}

Tom Siday, Michele Natrella, Jiang Wu, Huiyun Liu, Oleg Mitrofanov

Tom Siday, Michele Natrella, Jiang Wu, Huiyun Liu, Oleg Mitrofanov, "Resonant scattering probes for terahertz near-field microscopy," Proc. SPIE 10540, Quantum Sensing and Nano Electronics and Photonics XV, 1054020 (26 January 2018); doi: 10.1117/12.2290903

SPIE. Event: SPIE OPTO, 2018, San Francisco, California, United States 


\title{
Resonant scattering probes for terahertz near-field microscopy
}

\author{
Thomas Siday ${ }^{*}$, Michele Natrella, Jiang Wu, Huiyun Liu and Oleg Mitrofanov \\ Electronic and Electrical Engineering Department, University College London, London, UK
}

\begin{abstract}
We propose and demonstrate a scattering-type near-field probe, designed to increase the sensitivity of high-resolution scattering probe microscopy at terahertz $(\mathrm{THz})$ frequencies. For efficient scattering of $\mathrm{THz}$ radiation, the probe, fabricated from indium, is designed to resonate like a dipole antenna. Efficient excitation is achieved by integrating the probe with a radially polarized THz source. Using time-domain spectroscopy (TDS), we observe resonant enhancement of the scattered fields, and using aperture-type near-field microscopy, we see high field confinement at the scattering probe apex.
\end{abstract}

Keywords: Near-field microscopy, Terahertz imaging.

\section{INTRODUCTION}

While the high resolution of near-field microscopy in the THz range has allowed for detailed study of many phenomena, such as plasmonics ${ }^{1,2}$ and resonators ${ }^{3,4}$, the sensitivity of the technique is still limited, resulting in impractical experimental configurations with long integration times. This is especially true when a metal tip is used to scatter light. This configuration, known as scattering-type near-field microscopy uses a sharp, usually metallic tip, the apex of which, after being excited by a THz field, interacts with the surface of the sample being imaged. Information about this nearfield interaction is included within a propagating $\mathrm{THz}$ field scattered by the tip ${ }^{5}$.

It was recently shown at infra-red frequencies that a scattering probe with length similar to the operation wavelength can act as a resonant dipole antenna and improve the efficiency of the scattering process ${ }^{6}$. Presently, the probes used for scattering-type microscopy in the $\mathrm{THz}$ range tend to be atomic force microscope (AFM) cantilevers, or sharp needles attached to a quartz tuning fork ${ }^{7-9}$. These are much shorter, and much longer than the $\mathrm{THz}$ wavelength respectively, meaning they will not support a $\mathrm{THz}$ dipole resonance. Therefore, a new type of probe, with length 50-500 $\mu \mathrm{m}$ $(1 \mathrm{THz}=300 \mu \mathrm{m})$ is required to create resonant antenna probes in the THz range.

In this paper, we demonstrate a new $\mathrm{THz}$ near-field probe, fabricated from indium, designed to act as a resonant dipole antenna. We evaluate the $\mathrm{THz}$ field confinement at the apex of the indium tips using a collection-mode aperture probe, and, using THz TDS, measure the broadband scattering properties of the probes to evaluate their scattering efficiency.

Fabricating the indium tips is a simple process, using a small drop of liquid indium on the end of a soldering iron. Tapping the iron on the surface of a semiconductor and then quickly retracting the iron leaves a sharp conical indium tip, such as those shown in Fig. 1.

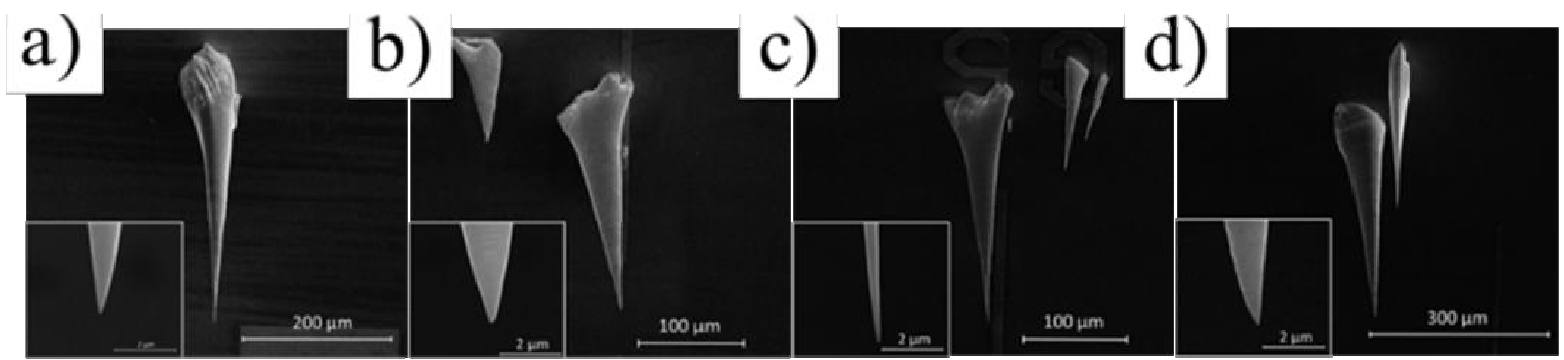

Figure 1. (a)-(d) scanning electron microscope images of a selection of indium tips.

Quantum Sensing and Nano Electronics and Photonics XV, edited by Manijeh Razeghi, Gail J. Brown,

Jay S. Lewis, Giuseppe Leo, Proc. of SPIE Vol. 10540, 1054020 - (c) 2018 SPIE

CCC code: $0277-786 X / 18 / \$ 18 \cdot$ doi: $10.1117 / 12.2290903$ 


\section{RADIAL EXCITATION}

Conventionally, a plane-polarized THz source, located in the far-field of a scattering tip is used for excitation, which produces a high $\mathrm{THz}$ field at the apex of the tip ${ }^{5}$. However, this technique results in large background fields, in particular those reflected from the surface of the sample under study. Alternatively, a radially polarized plasmonic mode can be generated on the surface of a conical scattering tip. As this mode travels toward the tip apex, the electromagnetic field is confined, with the maximum confinement determined by the radius of the tip apex ${ }^{10}$. Exploiting this phenomenon has proved difficult in the $\mathrm{THz}$ range due to a lack of radially polarized $\mathrm{THz}$ sources. While photoconductive antennas designed to emit a radial THz beam have been shown to couple to this radial plasmonic mode ${ }^{9,11,12}$, aligning the multiple components may prove difficult for scattering probe microscopy due to the spatially inhomogeneous polarization of the radially polarized $\mathrm{THz}$ beam. An alternative is to use a semiconductor $\mathrm{THz}$ source, where $\mathrm{THz}$ generation is localized to the optical excitation beam.

It was recently demonstrated that InAs can emit a radially-polarized $\mathrm{THz}$ beam ${ }^{13}$. When excited with femtosecond near infra-red (NIR) pulses $(\lambda=800 \mathrm{~nm})$, fast-moving charge carriers are accelerated radially on the InAs surface, the movement of which generates a co-propagating radial THz beam. This emission mechanism is visualized in Fig. 2(a). The indium tips can now be fabricated directly on the InAs source, trivializing the alignment of the NIR and THz beam to the base of the scattering probe.
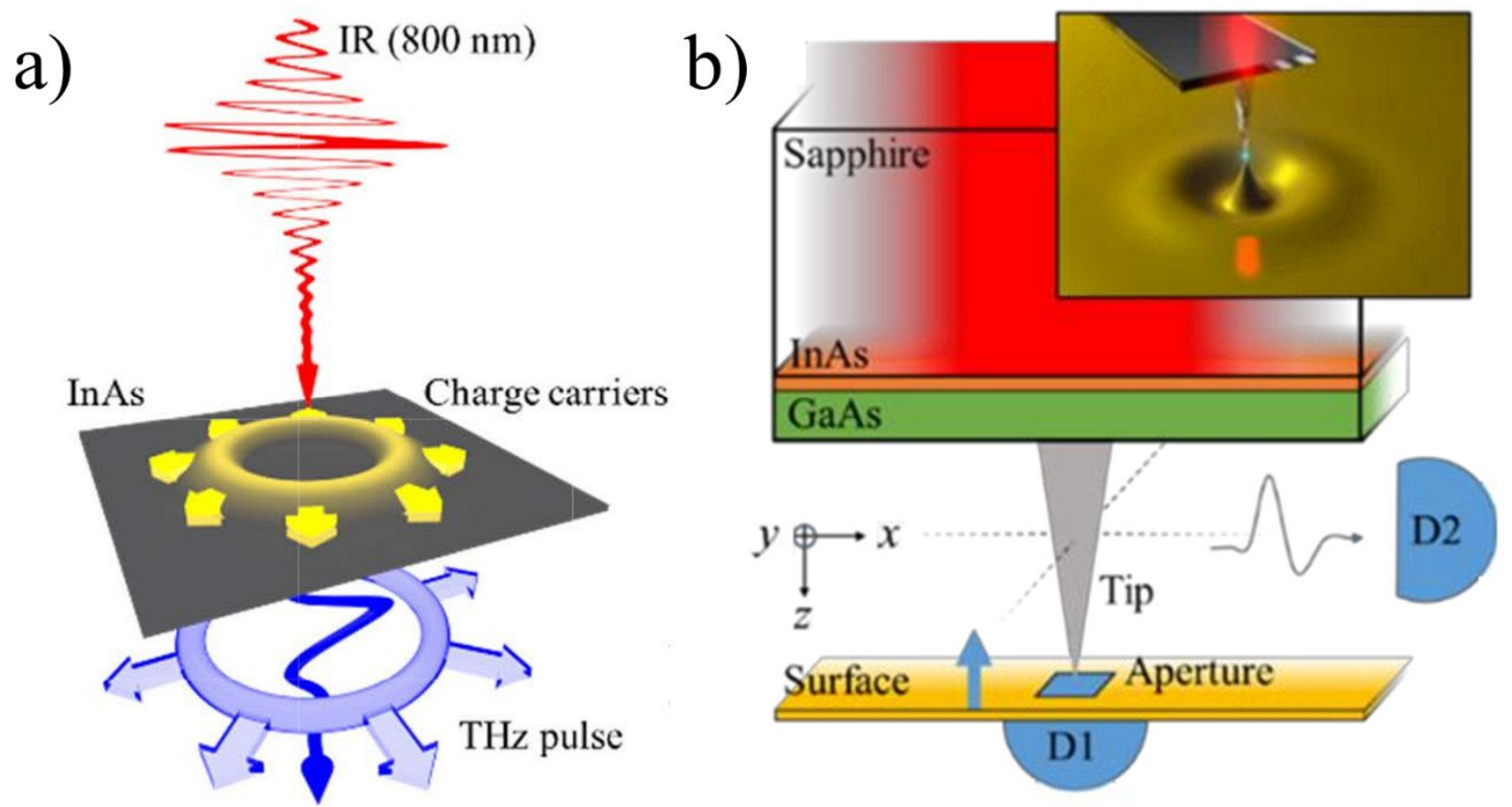

Figure 2. (a) A visualisation of the THz generation process in an InAs wafer. (b) Schematic of the experimental setup. The indium tip is raster-scanned in the x-y plane. The blue arrow shows the polarisation of surface waves on the aperture. Inset is a visualisation of the probe ${ }^{14}$.

\section{THZ FIELD AT THE INDIUM TIP APEX}

A collection-mode aperture probe, integrated into a THz TDS system was used to evaluate the THz field at the apex of the indium tip. While the spatial resolution of the aperture probe, at $3.9 \mu \mathrm{m}^{15}$ is lower than the predicted resolution of the indium tip $\left(\sim 100 \mathrm{~nm}^{5}\right)$, the aperture probe can still provide valuable information about the THZ field confinement at the tip apex. The experimental configuration is shown in Fig. 2(b). The apex of the indium tip is raster-scanned over the apex of the aperture probe in the $x-y$ plane, shown in the figure, to build up a $2 \mathrm{D}$ image of the $\mathrm{THz}$ electric field at an instant in time. An optical delay stage can be used to measure the THz field's variation in time. 

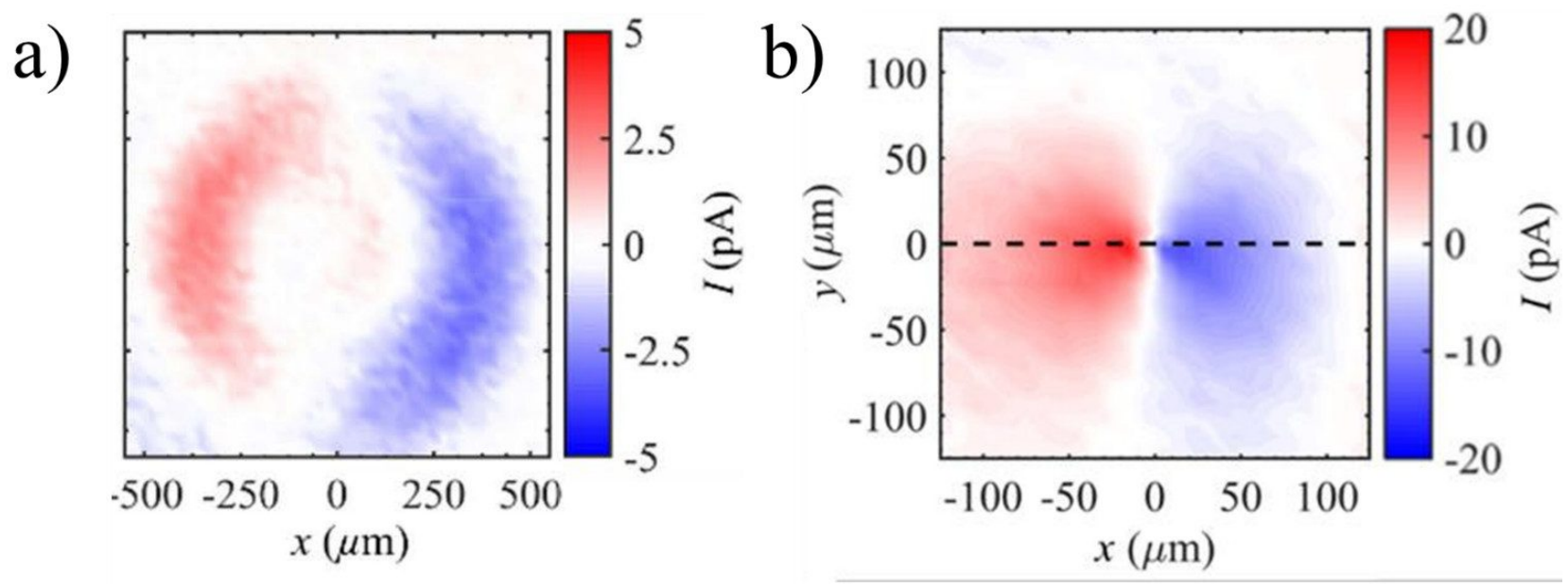

Figure 3. (a) Spatial field distribution measured by the aperture probe, of the emission of InAs on a plane $\sim 300 \mu \mathrm{m}$ from the InAs source. (b) Spatial field distribution measured by the aperture probe when $\mathrm{z} \sim 10 \mu \mathrm{m}$ from the apex of an indium tip $\sim 300 \mu \mathrm{m}$ long $^{14}$.

Figure 3. (a) shows the spatial field distribution of the $\mathrm{THz}$ emission from InAs, measured when no tip is present, showing the radial field distribution with the null central field. When an indium tip $\sim 300 \mu \mathrm{m}$ long is present, with the tip apex $z \sim 10 \mu \mathrm{m}$ from the aperture probe, the field distribution changes (Fig. 3(b)). Instead of the null field at the center of the beam, there is a maximum (note the spatial axis scales are not equal).

It is important to note that the aperture probe detects $\mathrm{THz}$ fields in different ways depending on the polarization of the incident $\mathrm{THz}$ field. For fields polarized parallel to the plane of the aperture, such as the emission from InAs, the field detected by the photoconductive antenna (PCA) behind the aperture is proportional to the time derivative of the incident field. As the PCA is configured to only detect fields polarized along a single axis $(x)$, the detected field is $E_{d e t}=d E_{x} / d t$. For fields polarized perpendicular to the plane of the aperture, such as surface waves, the spatial differential of the field is measured by the PCA, as the surface waves induce a potential across the two edges of the aperture, making the detected field $d E_{z} / d x$. This has been discussed in detail $\mathrm{in}^{1,16}$. The field detected when the indium tip is present is therefore a sum: $E_{d e t}=A\left(d E_{x} / d t\right)+B\left(d E_{z} / d x\right)$, where A and B are constants. This behavior of the aperture probe can be used to explain the minimum in the very center of Fig. 3(b). This is the point where the indium tip is directly over the aperture, such that the $E_{z}$ field is a maximum, and therefore $d E_{z} / d x$ is a minimum.

The tip is now brought within $1 \mu \mathrm{m}$ of the aperture. Again, the field maximum is centered at the location of the tip apex, though this time the field is confined to within the spatial resolution of the aperture probe $\left(3.9 \mu \mathrm{m}^{15}\right)$. This is a good indication that the indium tip is efficiently focusing the THz field generated by the InAs wafer to the tip apex. 
a)

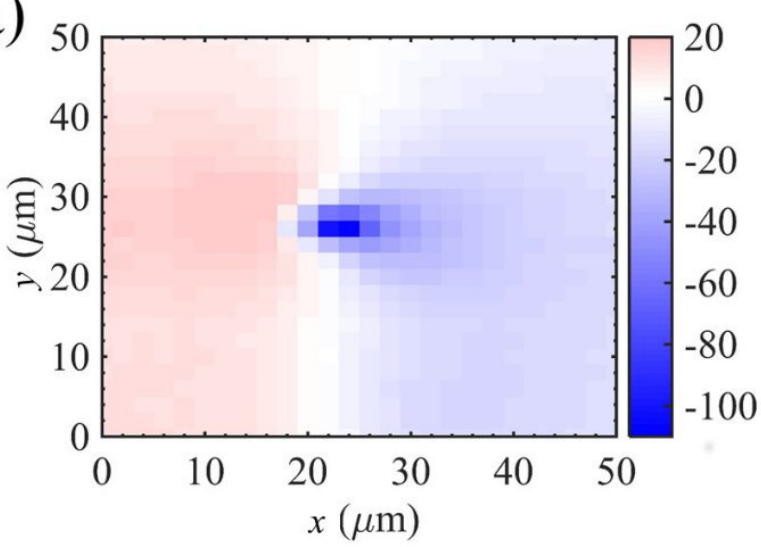

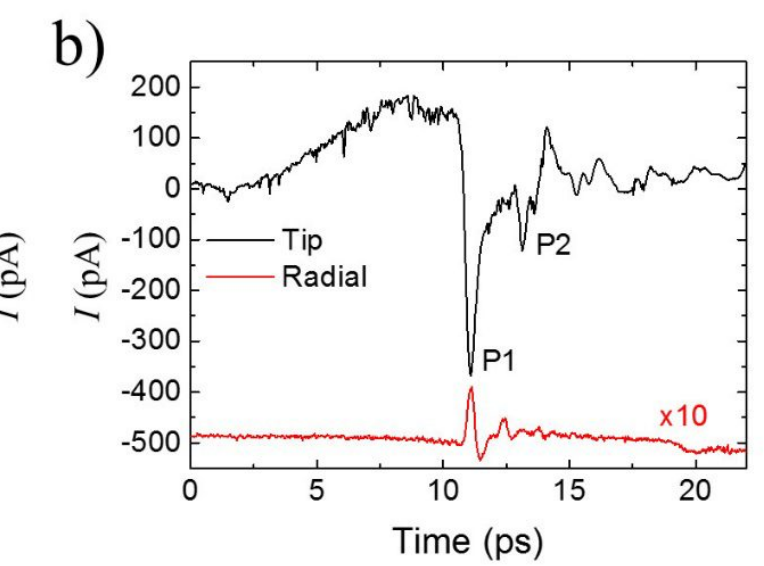

Figure 4. (a) Spatial field distribution when a $\sim 250 \mu \mathrm{m}$ long indium tip is $z<1 \mu \mathrm{m}$ from the aperture probe, measured at point P1 in (b). (b) Waveform measured when the indium tip is directly over the aperture. The emission of InAs (red) is magnified 10x on the $y$ axis for clarity ${ }^{14}$.

Positioning the indium tip over the aperture, we measured the THz field evolution in time. Figure 4(a) shows the waveform at the tip apex, compared to the emission of InAs. While the two waveforms arrive at the same time, they are not identical. The polarization difference at P1 is simply due to the non-homogeneous polarization of the InAs emission (see Fig. 3(a)). Peak P2 on the other hand does not appear in the InAs emission. We attribute this to a reflection along the $\sim 250 \mu \mathrm{m}$ long indium tip. The other features in the waveform measured at the tip apex most likely lie below the noise level in the measurement of The InAs emission.

\section{MEASURING THE SCATTERING EFFICIENCY}

While the field at the apex of the probe provides good indication of the strong field confinement possible with the indium tips, it does not provide any information about their THz scattering efficiency. To this end, we configured the THz TDS system to detect the field scattered by the indium tip, with detector D2 placed $\sim 5 \mathrm{~mm}$ from the tip, on the plane indicated in Fig. 2(b).

The scattering efficiency of the indium tip cannot be directly determined in this experiment, so we assume that the detected field when the tip is present is simply a sum of the field scattered by the tip, and the emission of the InAs: $E_{d e t, I}=E_{I n A s}+E_{t i p}$. Then, the THz field scattered by the indium tip can be isolated by measuring the THz emission when no tip is present $\left(E_{d e t, 2}=E_{I n A s}\right)$ and taking the difference; $E_{t i p}=E_{d e t, 1}-E_{d e t, 2}$. The scattering efficiency of the tip can therefore be approximated to be $E_{t i p} / E_{\text {InAs }}$.

For consistency with the near-field measurements, an aperture probe with a $10 \mu \mathrm{m}$ input aperture is used to detect the scattered field, as both probes have similar frequency filtering properties. Fig. 5(a) shows the measured THz waveforms, when there is no tip (black line), when a tip is added (red line) and the scattered field (blue line). The scattered field appears at a small time delay relative to the emission from InAs, which is expected, as the scattered field must first travel the length of the indium tip before travelling toward the aperture probe. Figure 5(b) shows the Fourier transformed spectrum of the emission from InAs (black line) and the scattering efficiency of the tip (green line). While the scattering efficiency curve shows the emission is spectrally different to the emission of InAs, there is no clear dipolar resonance. We anticipate that the peak at $\sim 1.2 \mathrm{THz}$ is due to a higher order dipolar resonance. 

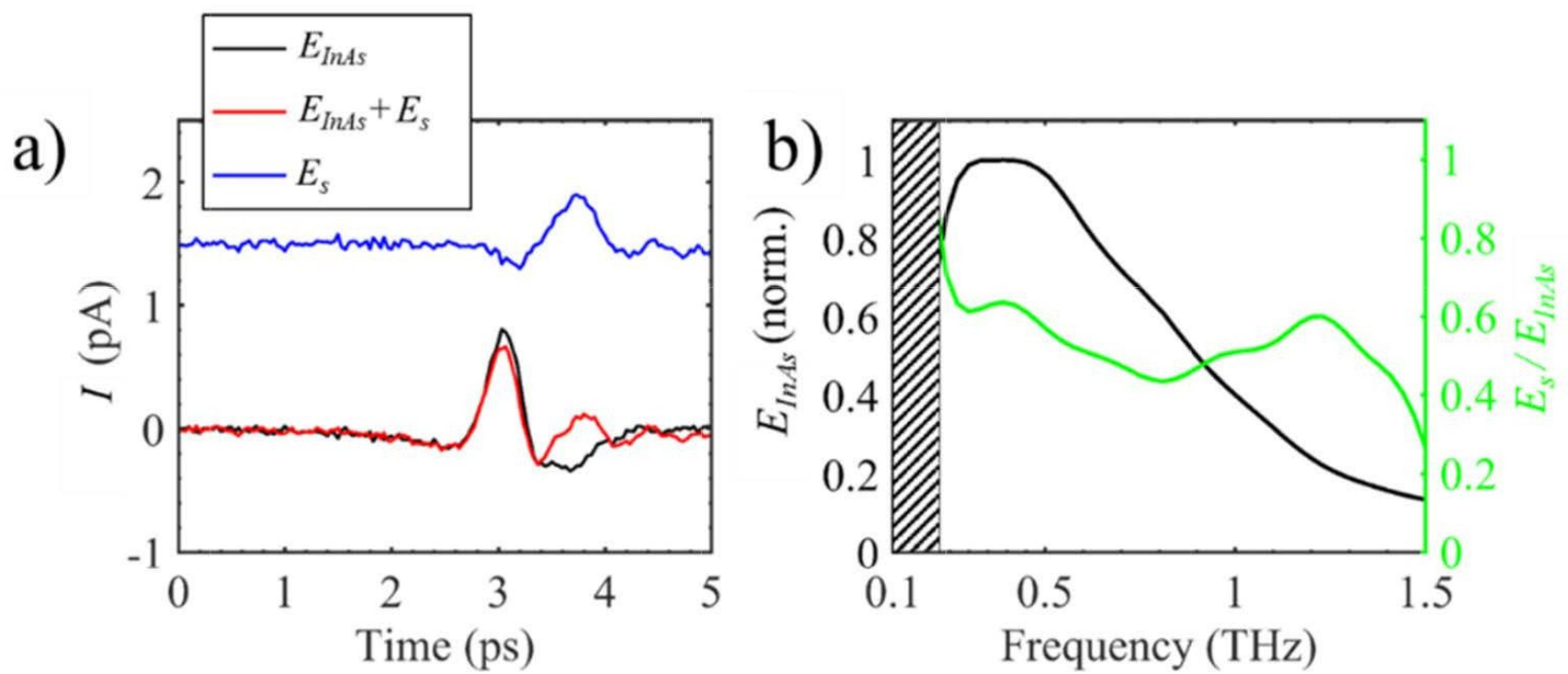

Figure 5. Indium tips configured for scattering, detected with a $10 \mu \mathrm{m}$ aperture probe. (a) Waveforms showing the $\mathrm{THz}$ emission of InAs (black line), when the indium tip is present (red line) and the scattered field (blue line). The scattered field is offset by $1.5 \mathrm{pA}$ on the $y$-axis for clarity. (b) Fourier transforms of the InAs emission in (a) (black line), multiplied by $1 / \mathrm{f}$ to account to the aperture transmission properties. The green line is the ratio $E_{s} / E_{\text {InAs }}$. Where the noise level is high, the plot is shaded ${ }^{14}$.

Whilst the $10 \mu \mathrm{m}$ aperture probe has similar spectral properties to the aperture probe used in the near-field measurements, sub $-\mathrm{THz}$ fields are attenuated strongly due to the $1 / f$ filtering of the aperture ${ }^{17}$. To eliminate this, the aperture probe is replaced with a PCA. Fig. 6 shows the same measurements as Fig 5, with the aperture probe replaced by the PCA. Note: the indium tips are not the same through the two experiments. This time the spectrum does show a strong peak in the scattering efficiency, at $0.3 \mathrm{THz}$.

The spectra shown in Fig 5(b) and 6(b) can be explained by considering the dipolar resonance of the indium tips. In this experiment, the fabricated tips were between $\sim 200$ and $400 \mu \mathrm{m}$ in length, and therefore they are expected to resonate below $1 \mathrm{THz}$. The low transmission of sub THz fields when using the aperture probe (Fig. 5) means it is likely that the dipolar resonance of the tip is lost below the noise level of the detector. This is confirmed by the spectra in Fig. 6(b), where there is a strong resonant peak centered at $\sim 0.3 \mathrm{THz}$. We estimate here the tip is $\sim 250 \mu \mathrm{m} \mathrm{long}{ }^{14}$. For resonant tips above $1 \mathrm{THz}$, we suggest tips with length $<150 \mu \mathrm{m}$ are used. 

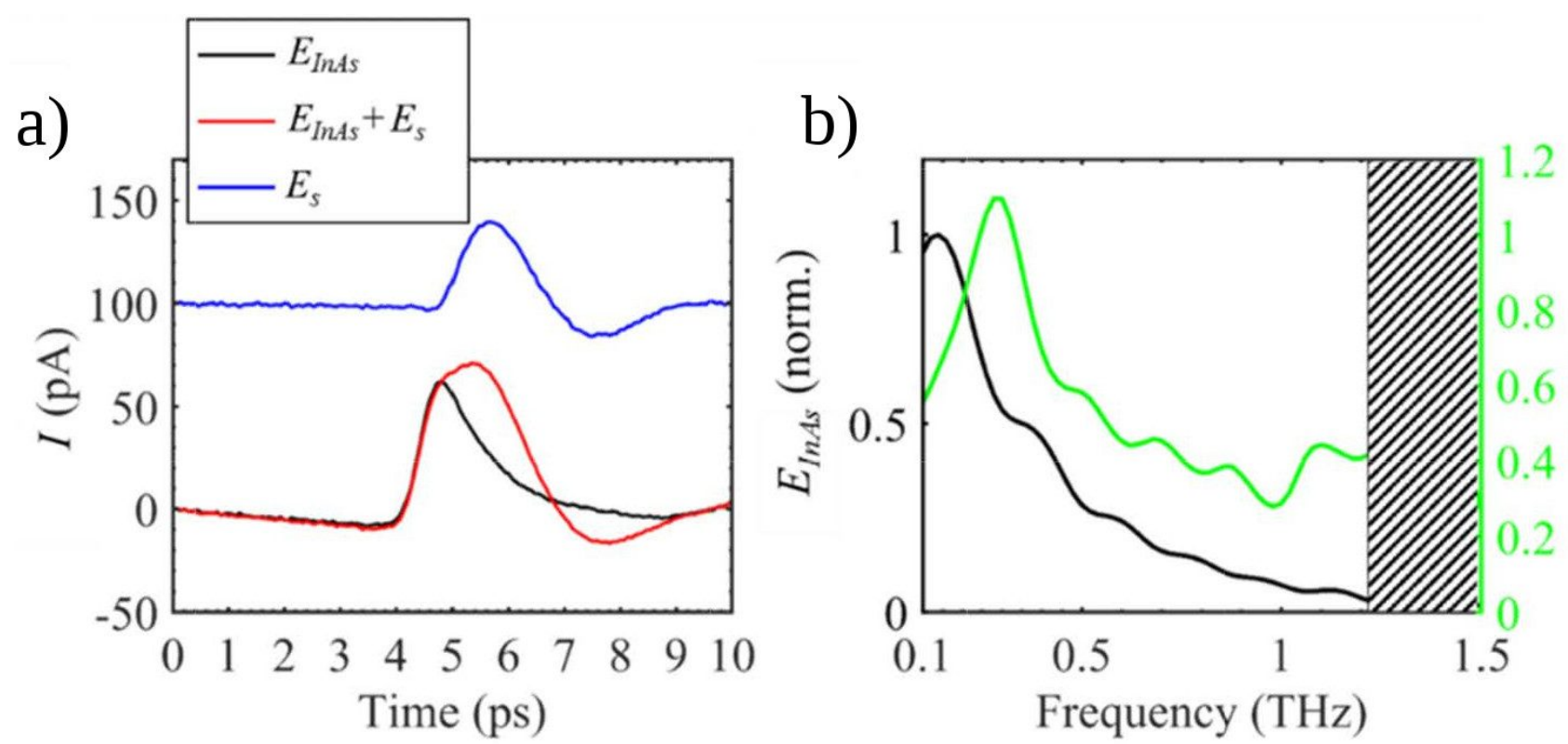

Figure 6. Waveforms and spectra where the aperture probe is replaced with a $\mathrm{PCA}^{14}$.

\section{CONCLUSIONS}

We have demonstrated a new scattering probe, designed to increase the efficiency of the scattering process by acting as a resonant $\mathrm{THz}$ dipole antenna. The indium tips taper to an apex of less than $100 \mathrm{~nm}$, which is on the order of the best spatial resolution possible in the $\mathrm{THz}$ range at present ${ }^{2}$. Tips are fabricated with molten indium on the end of a soldering iron, directly on the surface of a semiconductor $\mathrm{THz}$ source. The semiconductor source emits a radially polarized $\mathrm{THz}$ beam, which couples efficiently to a surface mode along the shaft of the indium tip. Experimental measurements in the near-field show that the tip has a strongly confined field at the apex. The scattering efficiency of the indium tip is measured, and the field scattered by the indium tip is indeed enhanced near the resonant frequency of the indium tip $(\sim 0.3 \mathrm{THz})$

\section{REFERENCES}

[1] Mueckstein, R. and Mitrofanov, O., "Imaging of terahertz surface plasmon waves excited on a gold surface by a focused beam," Opt. Express 19(4), 3212 (2011).

[2] Alonso-González, P., Nikitin, A. Y., Gao, Y., Woessner, A., Lundeberg, M. B., Principi, A., Forcellini, N., Yan, W., Vélez, S., Huber, A. J., Watanabe, K., Taniguchi, T., Casanova, F., Hueso, L. E., Polini, M., Hone, J., Koppens, F. H. L. and Hillenbrand, R., "Acoustic terahertz graphene plasmons revealed by photocurrent nanoscopy," Nat. Nanotechnol. 12(1), 31-35 (2017).

[3] Khromova, I., Kužel, P., Brener, I., Reno, J. L., Chung Seu, U.-C., Elissalde, C., Maglione, M., Mounaix, P. and Mitrofanov, O., "Splitting of magnetic dipole modes in anisotropic TiO2 micro-spheres," Laser Photonics Rev. 10(4), 681-687 (2016).

[4] Mitrofanov, O., Han, Z., Ding, F., Bozhevolnyi, S. I., Brener, I. and Reno, J. L., "Detection of internal fields in double-metal terahertz resonators," Appl. Phys. Lett. 110(6), 61109 (2017).

[5] Novotny, L. and Stranick, S. J., "Near-Field Optical Microscopy and Spectroscopy with Pointed Probes," Annu. Rev. Phys. Chem. 57(1), 303-331 (2006).

[6] Huth, F., Chuvilin, A., Schnell, M., Amenabar, I., Krutokhvostov, R., Lopatin, S. and Hillenbrand, R., "Resonant Antenna Probes for Tip-Enhanced Infrared Near-Field Microscopy," Nano Lett. 13(3), 1065-1072 (2013).

[7] Chen, H.-T., Kersting, R. and Cho, G., "Terahertz imaging with nanometer resolution," Appl. Phys. Lett. 83(15), 3009-3011 (2003). 
[8] Huber, A. J., Keilmann, F., Wittborn, J., Aizpurua, J. and Hillenbrand, R., "Terahertz Near-Field Nanoscopy of Mobile Carriers in Single Semiconductor Nanodevices6," Nano Lett. 8(11), 3766-3770 (2008).

[9] Astley, V., Mendis, R. and Mittleman, D. M., "Characterization of terahertz field confinement at the end of a tapered metal wire waveguide," Appl. Phys. Lett. 95(3), 31104 (2009).

[10] Stockman, M. I., "Nanofocusing of Optical Energy in Tapered Plasmonic Waveguides," Phys. Rev. Lett. 93(13), 137404 (2004).

[11] Zheng, Z., Kanda, N., Konishi, K. and Makoto, K.-G., "Efficient coupling of propagating broadband terahertz radial beams to metal wires," Opt. Express 21(9), 10642 (2013).

[12] Jeon, T.-I., Zhang, J. and Grischkowsky, D., "THz Sommerfeld wave propagation on a single metal wire,” Appl. Phys. Lett. 86(16), 161904 (2005).

[13] Mueckstein, R., Natrella, M., Hatem, O., Freeman, J., Graham, C., Renaud, C., Seeds, A., Linfield, E., Davies, A., Cannard, P., Robertson, M., Moodie, D. and Mitrofanov, O., "Near-Field Analysis of Terahertz Pulse Generation From Photo-Excited Charge Density Gradients," IEEE Trans. Terahertz Sci. Technol. 5(2), 260-267 (2015).

[14] Siday, T., Natrella, M., Wu, J., Liu, H. and Mitrofanov, O., "Resonant terahertz probes for near-field scattering microscopy,” Opt. Express 25(22), 27874-27885 (2017).

[15] Mitrofanov, O., Khromova, I., Siday, T., Thompson, R., Ponomarev, A., Brener, I. and Reno, J., "Near-Field Spectroscopy and Imaging of Subwavelength Plasmonic Terahertz Resonators," IEEE Trans. Terahertz Sci. Technol. 6(3), 382-388 (2016).

[16] Natrella, M., Mitrofanov, O., Mueckstein, R., Graham, C., Renaud, C. C. and Seeds, A. J., "Modelling of surface waves on a THz antenna detected by a near-field probe," Opt. Express 20(14), 16023-16031 (2012).

[17] Mitrofanov, O., Brener, I., Harel, R., Wynn, J., Pfeiffer, L., West, K. and Federici, J., "Terahertz near-field microscopy based on a collection mode detector," Appl. Phys. Lett. 77(22), 3496-3498 (2000). 